\title{
ECONOMIA CIRCULAR: CONCEITOS E APLICAÇÃO
}

\section{CIRCULAR ECONOMY: CONCEPTS AND APPLICATION}

\author{
Mario Roberto dos Santos ${ }^{1}$ \\ Fabio Ytoshi Shibao ${ }^{2}$ \\ Flavia Cristina da Silva ${ }^{3}$ \\ Resumo Abstract
}

O objetivo deste artigo foi verificar, nas edições do Journal of Cleaner Production, as pesquisas publicadas sobre economia circular e quais foram os objetivos e os principais achados dessas publicações. Foram encontrados 427 artigos e selecionados 40 artigos que mencionavam as palavras "circular economy" no título. Verificou-se a grande variedade de assuntos abordados, tais como modos de adoção; redução do uso de energia; uso na indústria da construção civil da água, de gesso, de serviços; avaliação de processos; comparação com os conceitos economia verde, bioeconomia, sustentabilidade; influência no design de produtos; indicadores de desempenho; origens; evolução; práticas empresariais; matriz orientativa para desmontagem de produtos; novas capacidades individuais; recuperação de valor; reutilização/reciclagem de equipamentos eletroeletrônicos; uso de cinzas de lodo de esgoto etc. É um tema vasto, com pesquisas em evolução, o que sugere que haverá muitas implicações nos modelos econômicos existentes e nos processos industriais atuais.

Palavras chaves: Economia circular. Meio ambiente. Resíduos.
The objective of this paper was to verify in the editions of the Journal of Cleaner Production, the published research on circular economics and what were the objectives and main findings of these publications. We found 427 articles and selected 40 articles that mentioned the words "circular economy" in the title. There was a great variety of topics addressed, such as: modes of adoption; reduction of energy use; use in the construction industry, water, plaster, services; process evaluation; comparison with the concepts green economy, bioeconomics, sustainability; influence on product design; performance indicators; origins; evolution; business practices; guiding matrix for product disassembly; new individual capacities; recovery of value; reuse / recycling of electrical and electronic equipment; use of sewage sludge ash; etc. It is a vast subject with evolving research, suggesting that there will be many implications for existing economic models and current industrial processes.

Keywords: Circular economy. Environment.Waste.

${ }^{1}$ Doutor em Administração pela Universidade Nove de Julho - UNINOVE. E-mail: mario.rsantos @ terra.com.br

${ }^{2}$ Professor e pesquisador do Programa de Mestrado Profissional de Administração em Gestão Ambiental e

Sustentabilidade (GeAS) da Universidade Nove de Julho - UNINOVE. E-mail: fabio.shibao@gmail.com

${ }^{3}$ Doutoranda do Programa de Pós-graduação em Administração de Empresas da Universidade Presbiteriana Mackenzie. Email: lacrisil@yahoo.com.br

Recebido em: 10 de março de 2019. Artigo aceito em 28 de dezembro de 2019. 


\section{Introdução}

A relação entre a indústria e o meio ambiente é crucial para o desempenho dos negócios industriais. Os impactos ambientais aumentaram gradativamente a pressão sobre as empresas industriais. Olhando para o início da revolução industrial, a produção em massa de mercadorias foi possibilitada por novos métodos de fabricação, resultando em produtos com alta disponibilidade e baixos custos. Consequentemente, devido às novas sociedades de consumo e ao crescimento vertiginoso da atividade industrial, as emissões para o meio ambiente, a geração de resíduos e os aterros sanitários tornaram-se cada vez mais numerosos. Além disso, em razão da crescente população mundial e, especialmente, do forte crescimento da classe média, estima-se que a demanda por recursos aumente rapidamente, indicando um crescente consumo de recursos naturais (LIEDER; RASHID, 2016).

Esse sistema linear de produção atual, com consumo e crescimento econômico cada vez maior está chegando ao fim, pois está ficando sem recursos, causando volatilidade de preços, incertezas e crises econômicas, ocasionando a deterioração dos ecossistemas (FISCHER; PASCUCCI, 2017).

Diversas abordagens surgiram ao longo das décadas, as quais tentaram lidar com esse fato de que os ecossistemas não podem sustentar a taxa de extração de matéria-prima e consumo de energia, nem a subsequente geração de resíduos resultante desse processo (FRANKLIN-JOHNSON; FIGGE; CANNING, 2016), sendo uma dessas abordagens o conceito de economia circular (EC).

Segundo Su et al. (2013), o conceito de EC foi levantado por dois economistas ambientais britânicos, Pearce e Turner (1990). Na publicação 'Economia de Recursos Naturais e Meio Ambiente', Pearce e Turner assinalaram que uma economia aberta tradicional era desenvolvida sem nenhum objetivo com a reciclagem e que tratava o meio ambiente como um reservatório de resíduos. No entanto, sob a primeira lei da termodinâmica, em que a energia total e a matéria permanecem constantes em um sistema fechado, o sistema aberto pode e deve ser convertido em um sistema circular ao considerar a relação entre o uso dos recursos e dos resíduos residuais. Em outras palavras, com os problemas ambientais existentes e a escassez de recursos, os autores sugeriram a necessidade de contemplar a terra como um sistema econômico fechado: aquele em que a economia e o meio ambiente não são relacionados por interligações lineares, mas por 
uma relação circular. Por intermédio de uma análise sobre a relação entre os sistemas econômico e natural, propuseram um circuito fechado de fluxos de materiais na economia, que foi denominado "economia circular".

A implementação efetiva do conceito de EC começou em 1996 na Alemanha, acompanhado de uma lei: "Ciclo Fechado de Substâncias e Lei de Gerenciamento de Resíduos". Essa lei estabeleceu o gerenciamento de resíduos de ciclo fechado e garantiu o descarte de resíduos compatível com o meio ambiente (SU et al., 2013).

A transição para uma economia circular, na qual o valor dos recursos é preservado, deve ser apoiada por políticas e decisões operacionais baseadas em evidências. Os métodos existentes usados para fornecer essa evidência (por exemplo, Life Cycle Assessment [LCA], Life Cycle Sustainability Assessment [LCSA], Cost-Benefit Analysis [CBA]) não são robustos o suficiente para abordar adequadamente a criação e a dissipação do valor sistêmico e multidimensional que abrange os domínios social, ambiental, econômico e técnico (IACOVIDOU, MILLWARD-HOPKINS et al., 2017).

Segundo Sehnem e Pereira (2019, p.35), “[...] a economia circular aumentou a sua capacidade de atrair a atenção de pesquisadores, gestores e governo[...]" e representa uma opção viável para transformar processos e fluxos de energia lineares em fluxos circulares e obter benefícios mais sustentáveis (SAAVEDRA et al., 2018), sendo um modelo alternativo que depende do aumento da eficiência de recursos e do desacoplamento do crescimento econômico do uso de recursos (ABU-GHUNMI et al., 2016).

O tema EC está no topo da agenda política, em particular na Europa, e por esse motivo espera-se que se promova o crescimento econômico criando novas empresas e oportunidades de emprego, economizando custos de materiais, amortecendo o preço da volatilidade, melhorando a segurança do fornecimento e, ao mesmo tempo, reduzindo as pressões e os impactos (KALMYKOVA; SADAGOPAN; ROSADO, 2018), pois propõe que o valores dos recursos extraídos e produzidos sejam mantidos em circulação por meio de cadeias produtivas integradas (SEHNEM; PEREIRA, 2019).

Ao promover a adoção de padrões de produção em um ciclo fechado em um sistema econômico, a EC visa aumentar a eficiência do uso de recursos, com foco especial nos resíduos urbanos e industriais, para alcançar um melhor equilíbrio e harmonia entre economia, meio ambiente e sociedade (GHISELLINI; CIALANI; ULGIATI, 2016). 
Kalmykova, Sadagopan e Rosado (2018) argumentaram que a disseminação da EC é dificultada porque o seu campo de aplicação é preenchido por abordagens divergentes. Além disso, nenhuma análise das estratégias de implementação de EC disponíveis e suas experiências de implementação foram suficientemente desenvolvidas, impedindo assim a sua implementação efetiva e colocando em risco os investimentos planejados para tal implementação.

Nesse contexto, o objetivo deste artigo foi verificar nas edições do Journal of Cleaner Production, as pesquisas publicadas sobre economia circular e quais foram os objetivos e os principais achados dessas publicações.

Este artigo está assim delineado: além desta introdução, a seção dois abordará a fundamentação teórica referente ao tema; a seção três apresenta os procedimentos metodológicos adotados; a seção quatro contempla os resultados e as discussões; na seção cinco apresentam-se as considerações finais.

\section{FUNDAMENTAÇÃO TEÓRICA}

A economia circular é, cada vez mais, vista como uma possível solução para abordar o desenvolvimento sustentável. Um sistema econômico que minimiza a entrada de recursos, a geração de resíduos e emissões, e vazamento de energia do sistema, espera mitigar os impactos negativos sem comprometer o crescimento e a prosperidade (GEISSDOERFER et al., 2018).

EC é um conceito promovido pela União Europeia (UE) e vários outros governos nacionais, entre os quais China, Japão, Canadá, Suécia e Finlândia, e Reino Unido, França, Holanda, bem como por várias empresas em todo o mundo. No entanto, o conceito de EC e sua prática têm sido quase exclusivamente desenvolvidos e conduzidos por profissionais, ou seja, decisores políticos, empresas, consultores, associações empresariais, fundações etc. (KORHONEN; HONKASALO; SEPPÄLÄ, 2018).

O conceito de EC está se difundindo, no entanto os críticos afirmam que esse conceito significa muitas coisas diferentes para pessoas diferentes. Kirchherr, Reike e Hekkert (2017) reuniram em sua pesquisa 114 definições de EC encontradas na literatura. Esses autores definiram EC como um sistema econômico que substitui o conceito de "fim de vida" por redução, reutilização alternativa, reciclagem e recuperação de materiais nos processos de produção/distribuição e consumo. Atua em nível micro (produtos, empresas, 
consumidores), nível meso (parques eco industriais) e macro (cidade, região, nação e além delas), com o objetivo de alcançar o desenvolvimento sustentável, criando, simultaneamente, qualidade ambiental, prosperidade e equidade social em benefício das gerações atuais e futuras. É possibilitado por novos modelos de negócios e consumidores responsáveis.

Embora com todo o interesse manifestado pela EC, os pesquisadores, particularmente no campo da gestão estratégica, ainda estão lutando com a falta de um quadro explicativo de como as empresas dispostas a praticar a EC deveriam adaptar seu modelo de negócios existente, ou criar um novo modelo. A atual representação, ou seja, aquela baseada em macro polos associados à extensão da vida útil dos produtos, sua redistribuição e reutilização, remanufatura e reciclagem não permitem distinguir diferentes modelos de negócios, ou seja, diferentes modos possíveis de adoção da EC pelas empresas. A literatura científica já estudou a EC sob várias perspectivas: (i) ecologia industrial, (ii) ciência ambiental, política e social e (iii) literatura sobre práticas de design de produto (URBINATI; CHIARONI; CHIESA, 2017).

Enquanto os termos EC e sustentabilidade estão cada vez mais ganhando força com a academia, com a indústria e os formuladores de políticas, as semelhanças e diferenças entre os dois conceitos permanecem ambíguas. A relação entre os conceitos não é explicitada na literatura, o que está confundindo seus contornos conceituais, além de restringir a eficácia do uso das abordagens na pesquisa e na prática. Isso tem implicações potencialmente prejudiciais para o avanço da ciência da sustentabilidade e a difusão de práticas baseadas nesses conceitos (GEISSDOERFER et al., 2017).

A implementação de uma EC exige que materiais, componentes e produtos (MCPs) sejam retidos na economia por mais tempo. Esta presunção requer a avaliação dos sistemas de recuperação de recursos a partir de resíduos (RRR), a montante e a jusante do ponto no qual os resíduos são gerados, a fim de permitir uma política sólida e os devidos processos de tomada de decisão. Para dar um passo significativo para o seu alcance, uma avaliação holística dos sistemas RRR com base no "valor complexo" multidimensional de MCPs, isto é, a soma holística de seus benefícios e impactos ambientais, econômicos, sociais e técnicos, e como eles são distribuídos ao longo do sistema e do tempo, torna-se cada vez mais necessária (IACOVIDOU; VELIS et al., 2017).

Revista Eletrônica Gestão e Serviços v.10, n. 2, pp. 2808 - 2826, Julho/Dezembro 2019.

ISSN Online: 2177-7284 e-mail: regs@metodista.br 
Nos últimos anos, o tema da EC está sendo amplamente explorado como um caminho possível para aumentar a sustentabilidade do sistema econômico. Reutilização, reparo e reciclagem estão se tornando atividades cruciais em muitos setores. Ao mesmo tempo, as empresas estão mostrando interesse crescente por esse novo modelo econômico. No entanto, o estado da arte mostra que ainda falta uma pesquisa profunda sobre avaliação e indicadores de EC, especialmente no nível micro (ELIA; GNONI; TORNESE, 2017).

Comparando-se EC com outros conceitos, tais como Economia Verde e Bioeconomia, apesar de suas suposições e estratégias de operacionalização diferentes, os conceitos são unidos pelo ideal comum de conciliar objetivos econômicos, ambientais e sociais. Os três conceitos são integrados na academia e na formulação de políticas como caminhos-chave de sustentabilidade (D'AMATO et al., 2017).

De los Rios e Charnley (2017) alertaram que uma variedade de novas capacidades é fundamental para projetar um futuro sustentável, e que estas variam desde o conhecimento mais profundo da composição dos materiais presentes nos produtos até a compreensão do comportamento social.

Portanto, estudar o conceito de EC nas pesquisas publicadas sobre o tema, e quais foram os objetivos e os principais achados dessas publicações, torna-se um desafio para a academia, no intuito de contribuir para a geração de insights que possibilite esse entendimento. A seguir são descritos os procedimentos metodológicos adotados nesta pesquisa.

\section{PROCEDIMENTOS METODOLÓGICOS}

O desenvolvimento e o crescimento da importância da ciência, tecnologia e inovação aumentaram as métricas sobre a produção intelectual e as informações científicas. Nesse sentido, o avanço das tecnologias de informação facilitou as pesquisas e a sua mensuração, principalmente em relação à bibliometria (MORETTI; CAMPANÁRIO, 2009).

Este estudo é caracterizado como descritivo, com abordagens qualitativas, por meio de análise de conteúdo dos artigos encontrados (BARDIN, 2009); e quantitativas, quanto ao dimensionamento dos dados.

Revista Eletrônica Gestão e Serviços v.10, n. 2, pp. 2808 - 2826, Julho/Dezembro 2019.

ISSN Online: 2177-7284 e-mail: regs@metodista.br 
Foi realizada uma pesquisa na base de dados do Journal of Cleaner Production, durante o mês de abril de 2018, com as palavras "circular economy" nas edições até dezembro de 2017. O objetivo foi verificar as pesquisas publicadas sobre o tema pelo periódico. O periódico está classificado pelo sistema Qualis 2013-2016 como A1. A escolha do periódico se deu porque, além da classificação Qualis, também havia disponibilidade de acesso.

A seguir serão apresentados os resultados obtidos nesta pesquisa.

\section{RESULTADOS}

Foram pesquisados artigos até o ano de 2017, inclusive, e dos tipos Review articles e Research articles. Encontrados 427 artigos, sendo 43 Review articles e 384 Research articles, no período 2008-2017. Foram lidos todos os títulos dos 427 artigos e selecionados os artigos que mencionavam as palavras "circular economy" no título. Restaram 40 artigos escolhidos para este trabalho.

\subsection{ARTIGOS SELECIONADOS}

O Quadro 1 mostra a relação dos artigos selecionados, ano de publicação, título e os respectivos autores.

\section{Quadro 1 - Artigos selecionados}

\begin{tabular}{|c|c|l|l|}
\hline $\mathbf{N}^{\mathbf{0}}$ & Ano & \multicolumn{1}{|c|}{ Título } & \multicolumn{1}{|c|}{ Autores } \\
\hline 1 & 2017 & Towards a new taxonomy of circular economy business models & $\begin{array}{l}\text { Urbinati, Chiaroni e } \\
\text { Chiesa }\end{array}$ \\
\hline 2 & 2017 & $\begin{array}{l}\text { Thermodynamic insights and assessment of the 'circular } \\
\text { economy }\end{array}$ & Cooper et al. \\
\hline 3 & 2017 & $\begin{array}{l}\text { Circular economy for the built environment: a research } \\
\text { framework }\end{array}$ & Pomponi e Moncaster \\
\hline 4 & 2017 & $\begin{array}{l}\text { Best practices for the management of end-of-life gypsum in a } \\
\text { circular economy }\end{array}$ & $\begin{array}{l}\text { Jiménez-Rivero e } \\
\text { García-Navarro }\end{array}$ \\
\hline 5 & 2017 & $\begin{array}{l}\text { Circular economy measures that boost the upgrade of an } \\
\text { aluminum industrial park }\end{array}$ & Han et al. \\
\hline 7 & 2017 & $\begin{array}{l}\text { Potential for circular economy in household WEEE } \\
\text { management }\end{array}$ & $\begin{array}{l}\text { The circular economy - a new sustainability paradigm? } \\
\text { Parajuly e Wenzel }\end{array}$ \\
\hline 8 & 2017 & $\begin{array}{l}\text { Skills and capabilities for a sustainable and circular economy: } \\
\text { the changing role of design }\end{array}$ & $\begin{array}{l}\text { De los Rios e } \\
\text { Charnley }\end{array}$ \\
\hline
\end{tabular}




\begin{tabular}{|c|c|c|c|}
\hline 9 & 2017 & $\begin{array}{l}\text { Integrated circular economy and education model to address } \\
\text { aspects of an energy-water-food nexus in a dairy facility and } \\
\text { local contexts }\end{array}$ & Kilk1s e K1lk1s \\
\hline 10 & 2017 & $\begin{array}{l}\text { Measuring circular economy strategies through index methods: } \\
\text { a critical analysis }\end{array}$ & Elia, Gnoni e Tornese \\
\hline 11 & 2017 & $\begin{array}{l}\text { Circular economy at the micro level: a dynamic view of } \\
\text { incumbents' struggles and challenges in the textile industry }\end{array}$ & Franco Mosquera \\
\hline 12 & 2017 & $\begin{array}{l}\text { Product design in the circular economy: users' perception of } \\
\text { end-of-life scenarios for electrical and electronic appliances }\end{array}$ & $\begin{array}{l}\text { Atlason, Giacalone e } \\
\text { Parajuly }\end{array}$ \\
\hline 13 & 2017 & $\begin{array}{l}\text { Integration of dismantling operations into a value recovery plan } \\
\text { for circular economy }\end{array}$ & $\begin{array}{l}\text { Cong, Zhao e } \\
\text { Sutherland }\end{array}$ \\
\hline 14 & 2017 & $\begin{array}{l}\text { Institutional incentives in circular economy transition: the case } \\
\text { of material use in the Dutch textile industry }\end{array}$ & Fischer e Pascucci \\
\hline 15 & 2017 & $\begin{array}{l}\text { A pathway to circular economy: developing a conceptual } \\
\text { framework for complex value assessment of resources } \\
\text { recovered from waste }\end{array}$ & $\begin{array}{l}\text { Iacovidou, Millward- } \\
\text { Hopkins et al. }\end{array}$ \\
\hline 16 & 2017 & $\begin{array}{l}\text { Comparative assessment of circular economy development in } \\
\text { China's four megacities: the case of Beijing, Chongqing, } \\
\text { Shanghai and Urumqi }\end{array}$ & Guo, Geng, Ren et al. \\
\hline 17 & 2017 & $\begin{array}{l}\text { Investigating public awareness on circular economy in western } \\
\text { China: A case of Urumqi Midong }\end{array}$ & Guo, Geng, Sterr et al. \\
\hline 18 & 2017 & $\begin{array}{l}\text { Institutional pressures, sustainable supply chain management, } \\
\text { and circular economy capability: empirical evidence from } \\
\text { Chinese eco-industrial park firms }\end{array}$ & Zeng et al. \\
\hline 19 & 2017 & $\begin{array}{l}\text { Metrics for optimizing the multi-dimensional value of } \\
\text { resources recovered from waste in a circular economy: a } \\
\text { critical review }\end{array}$ & Iacovidou, Velis et al. \\
\hline 20 & 2017 & $\begin{array}{l}\text { Evaluating the comprehensive benefit of eco-industrial parks } \\
\text { by employing multi-criteria decision making approach for } \\
\text { circular economy }\end{array}$ & Zhao, Zhao e Guo \\
\hline 21 & 2017 & $\begin{array}{l}\text { MFCA extension from a circular economy perspective: model } \\
\text { modifications and case study }\end{array}$ & Zhou et al. \\
\hline 22 & 2017 & $\begin{array}{l}\text { The need for better measurement and employee engagement to } \\
\text { advance a circular economy: lessons from biogen's "zero } \\
\text { waste" journey }\end{array}$ & $\begin{array}{l}\text { Veleva, Bodkin e } \\
\text { Todorova }\end{array}$ \\
\hline 23 & 2017 & $\begin{array}{l}\text { Green, circular, bio economy: a comparative analysis of } \\
\text { sustainability avenues }\end{array}$ & D'Amato et al. \\
\hline 24 & 2016 & $\begin{array}{l}\text { Resource duration as a managerial indicator for circular } \\
\text { economy performance }\end{array}$ & $\begin{array}{l}\text { Franklin-Johnson, } \\
\text { Figge e Canning }\end{array}$ \\
\hline 25 & 2016 & $\begin{array}{l}\text { Resource recovery from post-consumer waste: important } \\
\text { lessons for the upcoming circular economy }\end{array}$ & Singh e Ordoñez \\
\hline 26 & 2016 & $\begin{array}{l}\text { Towards circular economy implementation: a comprehensive } \\
\text { review in context of manufacturing industry }\end{array}$ & Lieder e Rashid \\
\hline 27 & 2016 & $\begin{array}{l}\text { Circular economy and the opportunity cost of not 'closing the } \\
\text { loop' of water industry: the case of Jordan }\end{array}$ & Abu-Ghunmi et al. \\
\hline 28 & 2016 & $\begin{array}{l}\text { A review on circular economy: the expected transition to a } \\
\text { balanced interplay of environmental and economic systems }\end{array}$ & $\begin{array}{l}\text { Ghisellini, Cialani e } \\
\text { Ulgiati }\end{array}$ \\
\hline 29 & 2016 & $\begin{array}{l}\text { Two life cycle assessment (LCA) based methods to analyze and } \\
\text { design complex (regional) circular economy systems. Case: } \\
\text { making water tourism more sustainable }\end{array}$ & $\begin{array}{l}\text { Scheepens, } \\
\text { Vogtländer e Brezet }\end{array}$ \\
\hline 30 & 2015 & $\begin{array}{l}\text { Strategies on implementation of waste-to-energy (WTE) supply } \\
\text { chain for circular economy system: a review }\end{array}$ & Pan et al. \\
\hline 31 & 2015 & $\begin{array}{l}\text { Product services for a resource-efficient and circular economy } \\
- \text { a review }\end{array}$ & Tukker \\
\hline
\end{tabular}

Revista Eletrônica Gestão e Serviços v.10, n. 2, pp. 2808 - 2826, Julho/Dezembro 2019. ISSN Online: 2177-7284 e-mail: regs@metodista.br 


\begin{tabular}{|l|l|l|l|}
32 & 2015 & $\begin{array}{l}\text { A case study of a phosphorus chemical firm's application of } \\
\text { resource efficiency and eco-efficiency in industrial metabolism } \\
\text { under circular economy }\end{array}$ & Ma et al. \\
\hline 33 & 2015 & Circular economy of a papermaking park in China: a case study & Li e Ma \\
\hline 34 & 2015 & $\begin{array}{l}\text { The possible use of sewage sludge ash (SSA) in the } \\
\text { construction industry as a way towards a circular economy }\end{array}$ & Smol et al. \\
\hline 36 & 2014 & $\begin{array}{l}\text { Quantitative assessment of industrial symbiosis for the } \\
\text { promotion of circular economy: a case study of the printed } \\
\text { circuit boards industry in China's Suzhou New District }\end{array}$ & Wen e Meng \\
\hline 37 & 2013 & $\begin{array}{l}\text { A reve study in Wu'an city } \\
\text { rhetoric to implementation }\end{array}$ & Ma et al. \\
\hline 38 & 2011 & $\begin{array}{l}\text { Ecological utilization of leather tannery waste with circular } \\
\text { economy model }\end{array}$ & Hu et al. \\
\hline 39 & 2010 & $\begin{array}{l}\text { Creating integrated business and environmental value within } \\
\text { the context of China's circular economy and ecological } \\
\text { modernization }\end{array}$ & Park, Sarkis e Wu \\
\hline 40 & 2009 & $\begin{array}{l}\text { A survey and analysis on public awareness and performance for } \\
\text { promoting circular economy in China: A case study from } \\
\text { Tianjin }\end{array}$ & Liu et al. \\
\hline
\end{tabular}

Fonte: Journal of Cleaner Production

A Gráfico 1 mostra a quantidade de artigos publicados por ano, no período 2009-2017.

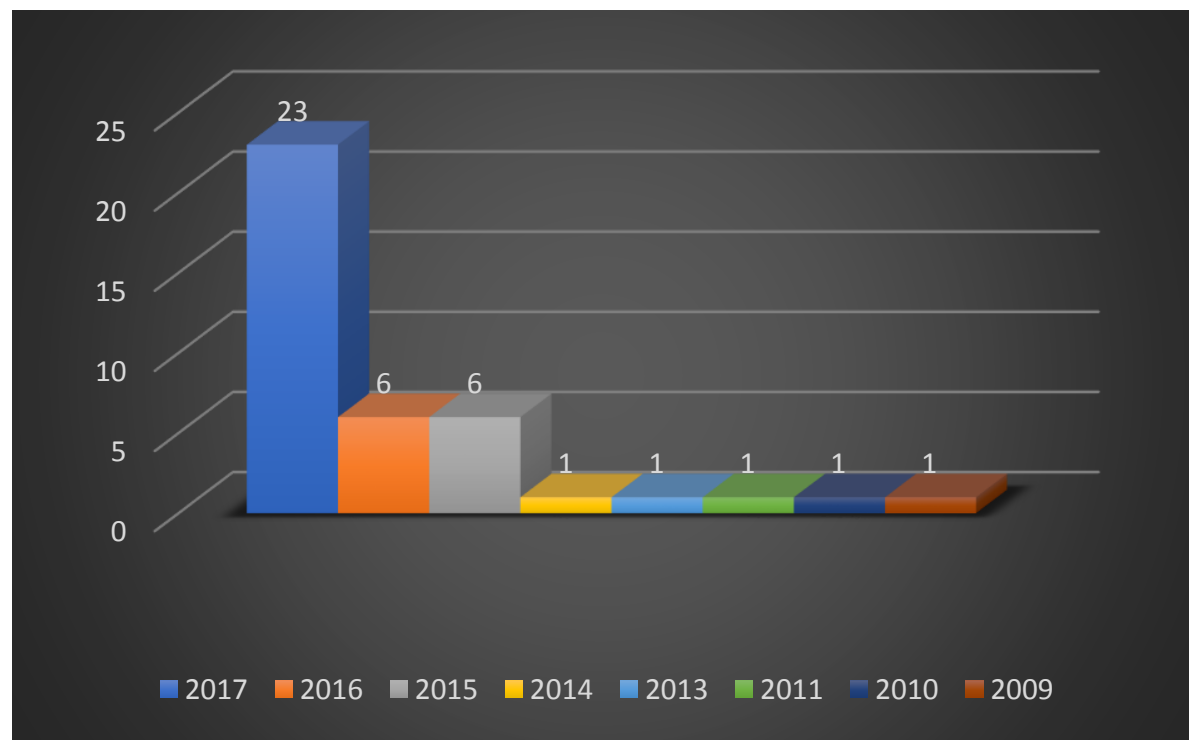

Gráfico 1 - Artigos publicados por ano no período 2009-2017

Fonte: Dados da pesquisa

O que se nota no Gráfico 1 é o crescimento do interesse de pesquisas sobre EC no periódico, sendo o ano de 2017 a maior quantidade do período, com 23 publicações, correspondendo a 57,5\% dos artigos publicados. 
A Tabela 1 mostra a quantidade de artigos publicados, por país ou região originários da pesquisa encontrados na pesquisa.

Tabela 1 - País ou região de autoria dos artigos

\begin{tabular}{c|c|c|c}
\hline País/Região & Quantidade & País/Região & Quantidade \\
\hline China & 14 & Alemanha & 1 \\
Reino Unido & 7 & Finlândia & 1 \\
Europa & 4 & França & 1 \\
Holanda & 4 & Jordânia & 1 \\
Itália & 3 & Taiwan & 1 \\
Suécia & 3 & Turquia & 1 \\
Dinamarca & 2 & & \\
\hline
\end{tabular}

Fonte: Dados da pesquisa

A Tabela 1 mostrou a grande quantidade de artigos publicados por autores chineses em relação aos demais países. Observa-se somente que, de acordo com o critério adotado, um artigo pode ter autores de diversos países e, também, às vezes, mencionada a região como foi definida no artigo, independentemente de a região abranger países já citados.

\subsection{AVALIAÇÃO DOS ARTIGOS}

Avaliando-se individualmente os artigos, quatro tiveram como objetivo a revisão da literatura: relação entre os conceitos de EC e de sustentabilidade (GEISSDOERFER et al., 2017); aspectos de escassez de recursos, geração de resíduos e vantagens econômicas (LIEDER; RASHID, 2016); principais características e perspectivas da EC: origens, princípios básicos, vantagens e desvantagens, modelagem e implementação nos diferentes níveis (micro, meso e macro) (GHISELLINI; CIALANI; ULGIATI, 2016). Destaca-se a pesquisa de Tukker (2015) sobre sistemas de serviços de produtos (Product Service Systems [PSS]), definida pelo autor como uma mistura de produtos tangíveis e serviços intangíveis projetados e combinados para que sejam capazes de atender às necessidades dos clientes finais. A pesquisa cita como as empresas implementaram, e quais são os principais fatores e questões para o sucesso.

Revista Eletrônica Gestão e Serviços v.10, n. 2, pp. 2808 - 2826, Julho/Dezembro 2019. ISSN Online: 2177-7284 e-mail: regs@metodista.br 
Seis artigos propuseram modelos e indicadores: (i) estrutura de referência para o monitoramento de estratégia de EC (ELIA; CHIARONI; CHIESA, 2017); (ii) métricas para avaliar a recuperação de recursos a partir dos resíduos gerados (IACOVIDOU; VELIS et al., 2017); (iii) taxonomia de modelos de negócios com base em duas grandes dimensões: proposta de valor ao cliente e interface; e rede de valor (URBINATI; CHIARONI; CHIESA, 2017); (iv) modelo para a prática de expanded zero waste, que inclui indicadores para medir os resultados e impactos das estratégias circulares de negócios (VELEVA; BODKIN; TODOROVA, 2017); (v) indicador para o desempenho da avaliação ambiental vinculado à EC (FRANKLIN-JOHNSON; FIGGE; CANNING, 2016); e (vi) aplicação do modelo Eco-costs Value Ratio (EVR) baseado em Avaliação do ciclo de vida (ACV) para analisar potenciais efeitos ambientais negativos empresariais (SCHEEPENS; VOGTLÄNDER; BREZET, 2016).

Dez artigos apresentaram os conceitos de EC aplicados em processos industriais e uso pós-consumo, seja por redução de consumo, seja por reutilização, reciclagem de resíduos, conforme apresenta o Quadro 2.

Uma pesquisa que se destaca é o artigo de Atlason, Giacalone e Parajuly (2017). Os autores verificaram se consumidores finais estão alinhados com o conceito de EC, em seguida, focaram em cenários após o final da vida útil (End-of-life [EoL]) de produtos eletroeletrônicos de uso doméstico e concluíram que os consumidores são favoráveis à reutilização de tais produtos, sugerindo que essa preferência está alinhada com o conceito de EC.

Quadro 2 - EC aplicada em processos industriais

\begin{tabular}{|c|l|l|}
\hline $\mathbf{N}^{\mathbf{0}}$ & \multicolumn{1}{|c|}{ Autores } & \multicolumn{1}{c|}{ Objeto } \\
\hline 1 & $\begin{array}{l}\text { Atlason, } \\
\text { Giacalone e } \\
\text { Parajuly 2017 }\end{array}$ & $\begin{array}{l}\text { Pesquisa sobre a destinação de produtos elétricos e eletrônicos pelos consumidores } \\
\text { finais e o alinhamento com o conceito de EC. }\end{array}$ \\
\hline 2 & $\begin{array}{l}\text { Cong, Zhao e } \\
\text { Sutherland, 2017 }\end{array}$ & $\begin{array}{l}\text { Matriz de desmontagens de produtos para formular a recuperação do valor do } \\
\text { produto no seu final da vida útil. }\end{array}$ \\
\hline 3 & $\begin{array}{l}\text { Cooper et al., } \\
2017\end{array}$ & $\begin{array}{l}\text { Uso de energia dentro dos conceitos de EC na União Europeia e Reino Unido, } \\
\text { como um dos fatores de potencial economia dessa energia. }\end{array}$ \\
\hline 4 & $\begin{array}{l}\text { Jiménez-Rivero } \\
\text { e García- } \\
\text { Navarro, 2017 }\end{array}$ & Melhores práticas para o uso de resíduos de gesso. \\
\hline 5 & $\begin{array}{l}\text { Parajuly e } \\
\text { Wenzel, 2017 }\end{array}$ & $\begin{array}{l}\text { Reutilização e reciclagem no gerenciamento de resíduos de equipamentos elétricos } \\
\text { e eletrônicos (WEEE) domésticos. }\end{array}$ \\
\hline
\end{tabular}




\begin{tabular}{|c|l|l|}
6 & $\begin{array}{l}\text { Pomponi e } \\
\text { Moncaster, 2017 }\end{array}$ & EC na construção civil de edifícios. \\
\hline 7 & $\begin{array}{l}\text { Abu-Ghunmi et } \\
\text { al., 2016 }\end{array}$ & $\begin{array}{l}\text { Estudar a dimensão econômica, a fim de estimar o custo de oportunidade de uma } \\
\text { indústria de água. }\end{array}$ \\
\hline 8 & $\begin{array}{l}\text { Singh e Ordoñez, } \\
2016\end{array}$ & $\begin{array}{l}\text { Análise de produtos desenvolvidos a partir de materiais descartados, } \\
\text { categorizando-os conforme a literatura de EC. }\end{array}$ \\
\hline 9 & Pan et al., 2015 & $\begin{array}{l}\text { Avaliação de tecnologias que usam resíduos para geração de energia (the waste-to- } \\
\text { energy [WTE]). }\end{array}$ \\
\hline 10 & Smol et al., 2015 & $\begin{array}{l}\text { Uso de cinzas de lodo de esgoto (sewage sludge ash [SSA]) para fabricação de } \\
\text { materiais de construção, matéria-prima para produção de cimento, agregados para } \\
\text { concreto e argamassa, e substituto de areia e / ou cimento. }\end{array}$ \\
\hline
\end{tabular}

Fonte: Dados da pesquisa

Os autores chineses foram os que mais publicaram, com 14 artigos, dentro do objeto aqui avaliado e mostraram o desenvolvimento da EC diante da evolução industrial chinesa, conforme mostra o Quadro 3, a seguir.

Quadro 3 - Artigos publicados por autores chineses no período

\begin{tabular}{|c|c|c|}
\hline $\mathbf{N}^{\mathbf{o}}$ & Autores & Objeto \\
\hline 1 & $\begin{array}{l}\text { Guo, Geng, Ren et } \\
\text { al., } 2017\end{array}$ & $\begin{array}{l}\text { Desenvolvimento da EC em quatro megacidades chinesas usando um sistema de } \\
\text { indicador unificado. }\end{array}$ \\
\hline 2 & $\begin{array}{l}\text { Guo, Geng, Sterr et } \\
\text { al., } 2017\end{array}$ & Comportamento dos consumidores em relação à EC. \\
\hline 3 & Han et al., 2017 & $\begin{array}{l}\text { Medidas de EC que impulsionam a atualização de um sistema industrial de } \\
\text { alumínio no Xinfa Industrial Park, China. }\end{array}$ \\
\hline 4 & Zeng et al., 2017 & $\begin{array}{l}\text { Relação entre pressões institucionais, gestão sustentável da cadeia de } \\
\text { suprimentos e capacidade de economia circular: evidências empíricas de } \\
\text { empresas chinesas de parques ecoindustriais. }\end{array}$ \\
\hline 5 & $\begin{array}{l}\text { Zhao, Zhao e Guo, } \\
2017\end{array}$ & $\begin{array}{l}\text { Proposta de uma estrutura híbrida para avaliar o benefício abrangente de } \\
\text { ecoparques industriais a partir da perspectiva da EC. }\end{array}$ \\
\hline 6 & Zhou et al., 2017 & $\begin{array}{l}\text { Combinar o modelo de contabilidade de custo de fluxo de materiais com o } \\
\text { princípio } 3 \mathrm{R} \text { e as características da indústria de alto consumo e poluição para o } \\
\text { cálculo de custos de processos de fabricação industrial. }\end{array}$ \\
\hline 7 & Li e Ma, 2015 & $\begin{array}{l}\text { Apresentação de um parque industrial chinês de fabricação de papel, que realiza } \\
\text { uma produção mais limpa e um desenvolvimento sustentável. }\end{array}$ \\
\hline 8 & Ma et al., 2015 & $\begin{array}{l}\text { Conjunto de ferramentas para ajudar os tomadores de decisão a resolver } \\
\text { problemas de eficiência ecológica e de recursos nas indústrias químicas chinesas. }\end{array}$ \\
\hline 9 & Wen e Meng, 2015 & $\begin{array}{l}\text { Combinação da abordagem de análise de fluxo de materiais com o indicador de } \\
\text { produtividade de recursos para avaliar a contribuição de ecoparques industriais } \\
\text { chineses para o desenvolvimento da EC. }\end{array}$ \\
\hline 10 & Ma et al., 2014 & $\begin{array}{l}\text { Avaliação da EC em uma siderúrgica chinesa, por meio de um sistema de } \\
\text { avaliação usado por essa indústria para estimar o desempenho da EC. }\end{array}$ \\
\hline 11 & Su et al., 2013 & $\begin{array}{l}\text { Um panorama de como a estratégia foi desenvolvida e implementada na China e, } \\
\text { em seguida, analisado o desempenho da EC em uma cidade e depois é } \\
\text { comparada com outras três cidades-piloto. }\end{array}$ \\
\hline
\end{tabular}




\begin{tabular}{|l|l|l|}
12 & Hu et al., 2011 & $\begin{array}{l}\text { Implementação do modelo de EC como uma nova forma de redução de consumo } \\
\text { de matérias-primas, água e energia na indústria do couro chinesa. }\end{array}$ \\
\hline 13 & $\begin{array}{l}\text { Park, Sarkis e Wu, } \\
2010\end{array}$ & $\begin{array}{l}\text { Desafios e oportunidades de como as empresas e organizações podem e serão } \\
\text { capazes de obter um melhor equilíbrio entre crescimento econômico e } \\
\text { administração ambiental no contexto de políticas de EC na China. }\end{array}$ \\
\hline 14 & Liu et al., 2009 & $\begin{array}{l}\text { Criar uma melhor compreensão da consciência pública e desempenho na } \\
\text { promoção de uma EC em Tianjin, China. }\end{array}$ \\
\hline
\end{tabular}

Fonte: Dados da pesquisa

Os autores chineses procuraram abordar, principalmente, as estratégias de implementação de EC na economia, mostrando as consequências, de uma forma geral, positivas para essa política de governo.

Nos seis artigos restantes, Franco Mosquera (2017) abordou as dificuldades de implementação da EC, avaliando barreiras e drivers para sua implementação no setor têxtil europeu; D'Amato et al. (2017) analisaram a diversidade dentro e entre os conceitos de EC, Economia Verde e Bioeconomia; Fischer e Pascucci (2017) avaliaram a colaboração entre empresas com novas formas de colaboração e o estímulo ao surgimento de novas instituições que privilegiam a sustentabilidade; De los Rios e Charnley (2017) analisaram as novas capacidades que são necessárias nos profissionais para o desenvolvimento de novos produtos; Iacovidou, Millward-Hopkins et al., (2017) verificaram como o valor dos recursos é criado, destruído e distribuído na recuperação desses recursos a partir de sistemas de resíduos. Uma aplicação na academia foi relatada por Kılk1s e Kilkıs (2017) sobre um sistema de EC em um curso universitário sobre política energética.

$\mathrm{O}$ que se verificou nas pesquisas é que as origens da EC estão, principalmente, enraizadas na economia ecológica e ambiental e na ecologia industrial. Na China, é promovida como um objetivo político nacional de cima para baixo, enquanto em outras áreas e países, tais como União Europeia, Japão e Estados Unidos da América (EUA), é uma ferramenta para projetar políticas de gestão de resíduos e ambientais de baixo para cima. A EC implica a adoção de padrões de produção mais limpa no nível da empresa, um aumento da responsabilidade e conscientização dos produtores e consumidores, o uso de tecnologias e de materiais renováveis (sempre que possível) e a adoção de políticas e ferramentas adequadas, claras e estáveis (GHISELLINI; CIALANI; ULGIATI,2016).

A implementação de práticas para uma EC transforma a maneira como as empresas fazem negócios, principalmente na indústria de manufatura. Requer uma 
transformação dos sistemas de produção e consumo, pois a abordagem tradicional de criação, de fabricação e de comércio de produtos deverá ser repensada (DE LOS RIOS; CHARNLEY, 2017).

O potencial de eficiência de recursos para reduzir os encargos ambientais e aumentar a resiliência à escassez de recursos é cada vez mais reconhecido globalmente, potencial esse difundido pelo conceito de EC (COOPER et al., 2017), e é tratado como uma solução para esses problemas (LIEDER; RASHID, 2016).

A transição para uma EC demanda mudanças ao longo da cadeia de valor, desde o design do produto até novos negócios e modelos de mercado, desde novas maneiras de transformar o desperdício em um recurso até novos modos de comportamento do consumidor, pois assim poderá manter o valor agregado do produto pelo maior tempo possível (SMOL et al., 2015).

Franklin-Johnson, Figge e Canning (2016) mencionaram que a EC está atraindo considerável interesse governamental e institucional em todo o mundo, mas, alertaram que, indiscutivelmente são as corporações e os respectivos gestores que são vitais para realizar as pretensões da EC.

Baseado no mapeamento efetuado foi feito um diagnóstico do perfil das publicações na área de EC no periódico Journal of Cleaner Production. O que essas informações implicam para a teoria? E para a prática da área empresarial e na área universitária? Considera-se que o mapeamento da configuração da publicação em uma área temática em específico, permite conhecer em que estágio se encontra a pesquisa naquele assunto. Isso gera oportunidades para novos estudos na área e mapeia o perfil de quem publica, das parcerias e dos vínculos.

\section{CONSIDERAÇÕES FINAIS}

O objetivo deste artigo foi verificar, nas publicações do Journal of Cleaner Production, as pesquisas publicadas sobre economia circular, quais foram os objetivos e os principais achados dessas publicações.

O que se constatou foi a grande variedade de assuntos abordados pelos artigos, tais como modos de adoção da EC; potencial de redução do uso global de energia; uso na indústria da construção civil, da água, de produtos de gesso; semelhanças e diferenças com sustentabilidade; avaliação de processos; comparação com os conceitos de economia 
verde e bioeconomia; influência no design de produtos; indicadores de desempenho; locais de origem/aplicação da EC; evolução do conceito; aplicação na área de serviços; práticas executadas em todos os níveis empresariais (níveis micro, meso e macro); matriz orientativa para desmontagem de produtos; novas capacidades individuais para projetos de produtos; recuperação de valor; reutilização/reciclagem de equipamentos eletroeletrônicos residenciais; uso de cinzas de lodo de esgoto; entre outros temas abordados.

Destacou-se a grande quantidade de artigos (14) publicados por autores chineses, correspondendo a 35\% dos artigos avaliados, mostrando a aplicação de EC na economia da China. Os autores avaliaram as aplicações de EC em regiões/cidades; em ecoparques industriais; indústrias químicas, couro, papel, siderurgia, alumínio; e comportamento do consumidor.

Como origem do tema e sua estratégia de implementação, a EC está vinculada a economia ecológica e ambiental e na ecologia industrial. Na China, é utilizada como um objetivo político nacional de cima para baixo, e na União Europeia, no Japão e nos EUA consolidou-se como uma ferramenta de políticas de gestão de resíduos e ambientais, aplicada de baixo para cima. A transição para a EC vem do envolvimento de todos os atores da sociedade e de sua capacidade de vincular e criar padrões adequados de colaboração e intercâmbio. As histórias de sucesso também apontaram para a necessidade de um retorno econômico do investimento, a fim de fornecer motivação adequada para empresas e investidores (GHISELLINI; CIALANI; ULGIATI, 2016).

Em razão dos resultados aqui apresentados, constata-se que é um tema extremamente vasto, com pesquisas em evolução, o que sugere que haverá muitas implicações nos modelos econômicos existentes e nos processos industriais atuais, portanto um campo ainda inexplorado de temas de pesquisa.

Uma das limitações do estudo decorre da utilização somente da base de dados do Journal of Cleaner Production, que, apesar de ser classificado como Qualis A1, limitou as conclusões, não permitindo a extensão dos resultados. Sugestão para pesquisas futuras é a utilização de diversas bases de dados incluindo outros periódicos.

\section{REFERÊNCIAS}

ABU-GHUNMI, D.; ABU-GHUNMI, L.; KAYAL, B.; BINO, A. Circular economy and the opportunity cost of not 'closing the loop' of water industry: the case of Jordan. 
Journal of Cleaner Production, v.131, p.228-236, 2016. https://doi.org/10.1016/j.jclepro.2016.05.043.

ATLASON, R. S.; GIACALONE, D.; PARAJULY, K. Product design in the circular economy: users' perception of end-of-life scenarios for electrical and electronic appliances. Journal of Cleaner Production, v.168, p.1059-1069, 2017. https://doi.org/10.1016/j.jclepro.2017.09.082.

Bardin, L. Análise de conteúdo, 5a ed. Lisboa: Edições 70. Lda., 2009.

CONG, L.; ZHAO, F.; SUTHERLAND, J. W. Integration of dismantling operations into a value recovery plan for circular economy. Journal of Cleaner Production, v.149, p.378-386, 2017. https://doi.org/10.1016/j.jclepro.2017.02.115.

COOPER, S. J. G.; GIESEKAM, J.; HAMMOND, G. P.; NORMAN, J. B.; SCOTT, K. Thermodynamic insights and assessment of the 'circular economy'. Journal of Cleaner Production, v.162, p.1356-1367, 2017. https://doi.org/10.1016/j.jclepro.2017.06.169.

D'AMATO, D.; DROSTE, N.; ALLEN, B.; KETTUNEN, M.; L€AHTINEN, K.; KORHONEN, J.; LESKINEN, P.; MATTHIES, B. D.; TOPPINEN, A. Green, circular, bio economy: a comparative analysis of sustainability avenues. Journal of Cleaner Production, v.168, p.716-734, 2017. https://doi.org/10.1016/j.jclepro.2017.09.053.

DE LOS RIOS, I. C.; CHARNLEY, F. J. S. Skills and capabilities for a sustainable and circular economy: the changing role of design. Journal of Cleaner Production, v.160, p.109-122, 2017. https://doi.org/10.1016/j.jclepro.2016.10.130.

ELIA, V.; GNONI, M. G.; TORNESE, F. Measuring circular economy strategies through index methods: a critical analysis. Journal of Cleaner Production, v.142, Part 4, p.2741-2751, 2017. https://doi.org/10.1016/j.jclepro.2016.10.196.

FISCHER, A.; PASCUCCI, S. Institutional incentives in circular economy transition: the case of material use in the Dutch textile industry. Journal of Cleaner Production, v.155, Part 2, p.17-32, 2017. https://doi.org/10.1016/j.jclepro.2016.12.038.

FRANCO MOSQUERA, M. A. Circular economy at the micro level: a dynamic view of incumbents' struggles and challenges in the textile industry. Journal of Cleaner Production, v.168, p.833-845, 2017. https://doi.org/10.1016/j.jclepro.2017.09.056.

FRANKLIN-JOHNSON, E.; FIGGE, F.; CANNING, L. Resource duration as a managerial indicator for circular economy performance. Journal of Cleaner Production, v.133, p.589-598, 2016. https://doi.org/10.1016/j.jclepro.2016.05.023.

GEISSDOERFER, M.; MORIOKA, S. N.; CARVALHO, M. M.; EVANS, S. Business models and supply chains for the circular economy. Journal of Cleaner Production, v.790, p.712-721, 2018. https://doi.org/10.1016/j.jclepro.2018.04.159.

GEISSDOERFER, M.; SAVAGET, P.; BOCKEN, N. M. P. HULTINK, E. J. The circular economy e a new sustainability paradigm? Journal of Cleaner Production, v.143, p.757-768, 2017. https://doi.org/10.1016/j.jclepro.2016.12.048.

GHISELLINI, P.; CIALANI, C.; ULGIATI, S. A review on circular economy: the expected transition to a balanced interplay of environmental and economic systems.

Journal of Cleaner Production, v.114, p.11-32, 2016.

https://doi.org/10.1016/j.jclepro.2015.09.007.

Revista Eletrônica Gestão e Serviços v.10, n. 2, pp. 2808 - 2826, Julho/Dezembro 2019.

ISSN Online: 2177-7284 e-mail: regs@metodista.br 
GUO, B.; GENG, Y.; REN, J.; ZHU, L.; STERR, T. Comparative assessment of circular economy development in China's four megacities: the case of Beijing, Chongqing, Shanghai and Urumqi. Journal of Cleaner Production, v.162, p.234-246, 2017. https://doi.org/10.1016/j.jclepro.2017.06.061.

GUO, B.; GENG, Y.; STERR, T.; ZHU, Q.; LIU, Y. Investigating public awareness on circular economy in western China: A case of Urumqi Midong. Journal of Cleaner Production, v.142, Part 4, p.2177-2186, 2017. https://doi.org/10.1016/j.jclepro.2016.11.063.

HAN, F.; LIU, Y.; LIU, W.; CUI, Z. Circular economy measures that boost the upgrade of an aluminum industrial park. Journal of Cleaner Production, v.168, p.1289-1296, 2017. https://doi.org/10.1016/j.jclepro.2017.09.115.

HU, J.; XIAO, Z.; ZHOU, R.; DENG, W.; MA, S. Ecological utilization of leather tannery waste with circular economy model. Journal of Cleaner Production, v.19, n.2-3, p.221-228, 2011. https://doi.org/10.1016/j.jclepro.2010.09.018.

IACOVIDOU, E.; MILLWARD-HOPKINS, J.; BUSCH, J.; PURNELL, P.; VELIS, C. A.; HAHLADAKIS, J. N.; ZWIRNER, O.; BROWN, A. A pathway to circular economy: developing a conceptual framework for complex value assessment of resources recovered from waste. Journal of Cleaner Production, v.168, p.1279-1288, 2017. https://doi.org/10.1016/j.jclepro.2017.09.002.

IACOVIDOU, E.; VELIS, C. A.; PURNELL, P.; ZWIRNER, O.; BROWN, A.; HAHLADAKIS, J. N.; MILLWARD-HOPKINS, J.; WILLIAMS, P. T. Metrics for optimizing the multidimensional value of resources recovered from waste in a circular economy: a critical review. Journal of Cleaner Production, v.166, p.910-938, 2017. https://doi.org/10.1016/j.jclepro.2017.07.100.

JIMÉNEZ-RIVERO, A.; GARCÍA-NAVARRO, J. Best practices for the management of end-of-life gypsum in a circular economy. Journal of Cleaner Production, v.167, p.1335-1344, 2017. https://doi.org/10.1016/j.jclepro.2017.05.068.

KALMYKOVA, Y.; SADAGOPAN, M.; ROSADO, L. Circular economy - from review of theories and practices to development of implementation tools. Resources, Conservation and Recycling, v.135, p.190-201, 2018.

https://doi.org/10.1016/j.resconrec.2017.10.034.

KILKIS, S.; KILKIS, B. Integrated circular economy and education model to address aspects of an energy-water-food nexus in a dairy facility and local contexts. Journal of Cleaner Production, v.167, p.1084-1098, 2017.

https://doi.org/10.1016/j.jclepro.2017.03.178.

KIRCHHERR, J.; REIKE, D.; HEKKERT, M. Conceptualizing the circular economy: an analysis of 114 definitions. Resources, Conservation and Recycling, v.127, p.221232, 2017. https://doi.org/10.1016/j.resconrec.2017.09.005.

KORHONEN, J.; HONKASALO, A.; SEPPÄLÄ, J. Circular economy: the concept and its limitations. Ecological Economics, v.143, p.37-46, 2018.

https://doi.org/10.1016/j.ecolecon.2017.06.041.

Revista Eletrônica Gestão e Serviços v.10, n. 2, pp. 2808 - 2826, Julho/Dezembro 2019.

ISSN Online: 2177-7284 e-mail: regs@metodista.br 
LI, Y.; MA, C. Circular economy of a papermaking park in China: a case study. Journal of Cleaner Production, v.92, p.65-74, 2015. https://doi.org/10.1016/j.jclepro.2014.12.098.

LIEDER, M.; RASHID, A. Towards circular economy implementation: a comprehensive review in context of manufacturing industry. Journal of Cleaner Production, v.115, p.36-51, 2016. https://doi.org/10.1016/j.jclepro.2015.12.042.

LIU, Q.; LI, H. M.; ZUO, X. L.; ZHANG, F. F; WANG, L. A survey and analysis on public awareness and performance for promoting circular economy in China: a case study from Tianjin. Journal of Cleaner Production, v.17, n.2, p.265-270, 2009. https://doi.org/10.1016/j.jclepro.2008.06.003.

MA, S.; HU, S.; CHEN, D.; ZHU, B. A case study of a phosphorus chemical firm's application of resource efficiency and eco-efficiency in industrial metabolism under circular economy. Journal of Cleaner Production, v.87, p.839-849, 2015. https://doi.org/10.1016/j.jclepro.2014.10.059.

MA, S. H.; WEN, Z. G.; CHEN, J. N.; ZHI-CHAO WEN, Z. C. Mode of circular economy in China's iron and steel industry: a case study in Wu'an city. Journal of Cleaner Production, v.64, p.505-512, 2014. https://doi.org/10.1016/j.jclepro.2013.10.008.

MORETTI, S. L. A.; CAMPANÁRIO, M. A. A produção intelectual brasileira em responsabilidade social empresarial - RSE sob a ótica da bibliometria. Revista de Administração Contemporânea, v.13, n.spe, p.68-86, 2009. http://dx.doi.org/10.1590/S1415-65552009000500006.

PAN, S. Y.; DU, M. A.; HUANG, I. T.; LIU, I. H.; CHIANG, P. C. Strategies on implementation of waste-to-energy (WTE) supply chain for circular economy system: a review. Journal of Cleaner Production, v.108, Part A, p.409-421, 2015. https://doi.org/10.1016/j.jclepro.2015.06.124.

PARAJULY, K.; WENZEL, H. Potential for circular economy in household WEEE management. Journal of Cleaner Production, v.151, p.272-285, 2017. https://doi.org/10.1016/j.jclepro.2017.03.045.

PARK, J.; SARKIS, J.; WU, Z. Creating integrated business and environmental value within the context of China's circular economy and ecological modernization. Journal of Cleaner Production, v.18, n.15, p.1494-1501, 2010.

https://doi.org/10.1016/j.jclepro.2010.06.001.

POMPONI, F.; MONCASTER, A. Circular economy for the built environment: a research framework. Journal of Cleaner Production, v.143, p.710-718, 2017. https://doi.org/10.1016/j.jclepro.2016.12.055.

SAAVEDRA, Y. M. B.; IRITANI, D. R.; PAVAN, A. L. R.; OMETTO, A. R. Theoretical contribution of industrial ecology to circular economy. Journal of Cleaner Production, v.170, p.1514-1522, 2018. https://doi.org/10.1016/j.jclepro.2017.09.260.

SCHEEPENS, A. E.; VOGTLÄNDER, J. G.; BREZET, J. C. Two life cycle assessment (LCA) based methods to analyze and design complex (regional) circular economy systems. Case: making water tourism more sustainable. Journal of Cleaner Production, v.114, p.257-268, 2016. https://doi.org/10.1016/j.jclepro.2015.05.075.

Revista Eletrônica Gestão e Serviços v.10, n. 2, pp. 2808 - 2826, Julho/Dezembro 2019. ISSN Online: 2177-7284 e-mail: regs@ metodista.br 
SEHNEM, S.; PEREIRA, S. C. F. Rumo à economia circular: sinergia existente entre as definições conceituais correlatas e apropriação para a literatura brasileira. RECADM Revista Eletrônica de Ciência Administrativa, v.18, n.1, p.35-62, 2019. http://dx.doi.org/10.21529/RECADM.2019002.

SINGH, J.; ORDOÑEZ, I. Resource recovery from post-consumer waste: important lessons for the upcoming circular economy. Journal of Cleaner Production, v.134, Part A, p.342-353, 2016. https://doi.org/10.1016/j.jclepro.2015.12.020.

SMOL, M.; KULCZYCKA, J.; HENCLIK, A.; GORAZDA, K.; WZOREK, Z. The possible use of sewage sludge ash (SSA) in the construction industry as a way towards a circular economy. Journal of Cleaner Production, v.95, p.45-54, 2015. https://doi.org/10.1016/j.jclepro.2015.02.051.

SU, B.; HESHMATI, A.; GENG, Y.; YU, X. A review of the circular economy in China: moving from rhetoric to implementation. Journal of Cleaner Production, v.42, p.215-227, 2013. https://doi.org/10.1016/j.jclepro.2012.11.020.

TUKKER, A. Product services for a resource-efficient and circular economy - a review. Journal of Cleaner Production, v.97, p.76-91, 2015. https://doi.org/10.1016/j.jclepro.2013.11.049.

URBINATI, A.; CHIARONI, D.; CHIESA, V. Towards a new taxonomy of circular economy business models. Journal of Cleaner Production, v.168, p.487-498, 2017. https://doi.org/10.1016/j.jclepro.2017.09.047.

VELEVA, V.; BODKIN, G.; TODOROVA, S. The need for better measurement and employee engagement to advance a circular economy: lessons from biogen's “zero waste" journey. Journal of Cleaner Production, v.154, p.517-529, 2017. https://doi.org/10.1016/j.jclepro.2017.03.177.

WEN, Z.; MENG, X. Quantitative assessment of industrial symbiosis for the promotion of circular economy: a case study of the printed circuit boards industry in China's Suzhou New District. Journal of Cleaner Production, v.90, p.211-219, 2015. https://doi.org/10.1016/j.jclepro.2014.03.041.

ZENG, H.; CHEN, X.; XIAO, X.; ZHOU, Z. Institutional pressures, sustainable supply chain management, and circular economy capability: empirical evidence from Chinese eco-industrial park firms. Journal of Cleaner Production, v.155, Part 2, p.54-65, 2017. https://doi.org/10.1016/j.jclepro.2016.10.093.

ZHAO, H.; ZHAO, H.; GUO, S. Evaluating the comprehensive benefit of eco-industrial parks by employing multi-criteria decision making approach for circular economy. Journal of Cleaner Production, v.142, Part 4, p.2262-2276, 2017. https://doi.org/10.1016/j.jclepro.2016.11.041.

ZHOU, Z.; ZHAO, W.; CHEN, X.; ZENG, H. MFCA extension from a circular economy perspective: model modifications and case study. Journal of Cleaner Production, v.149, p.110-125, 2017. https://doi.org/10.1016/j.jclepro.2017.02.049. 